\title{
Flow Modulates Myogenic Responses in Isolated Microperfused Rabbit Afferent Arterioles Via Endothelium-derived Nitric Oxide
}

\author{
Luis A. Juncos, Jeffrey Garvin, Oscar A. Carretero, and Sadayoshi Ito \\ Hypertension and Vascular Research Division, Department of Internal Medicine and Heart and Vascular Institute, Henry Ford Hospital, \\ Detroit, Michigan 48202
}

\begin{abstract}
Flow may be a physiological stimulus of the endothelial release of nitric oxide (NO) and prostaglandins (PGs). We tested the hypothesis that pressure-induced constriction of the glomerular afferent arteriole (Af-Art) is modulated by luminal flow via endothelial production of NO. We microdissected the terminal segment of an interlobular artery together with two Af-Arts, their glomeruli (GL) and efferent arterioles (Ef-Art). The two Af-Arts were perfused simultaneously from the interlobular artery, while one Ef-Art was occluded. Since the arteriolar perfusate contained $5 \%$ albumin, oncotic pressure built up in the glomerulus with the occluded Ef-Art and opposed the force of filtration, resulting in little or no flow through the corresponding Af-Art. Thus this preparation allowed us to observe free-flow and no-flow Af-Arts simultaneously during stepwise 30-mmHg increases in intraluminal pressure (from 30 to $120 \mathrm{mmHg}$ ). Pressureinduced constriction was weaker in free-flow than no-flow Af-Arts, with the luminal diameter decreasing by $11.1 \pm 1.7$ and $25.6 \pm 2.3 \%(n=30)$, respectively, at $120 \mathrm{mmHg}$. To examine whether flow modulates myogenic constriction through endothelium-derived NO and/or PGs, we examined pressure-induced constriction before and after $(a)$ disruption of the endothelium, $(b)$ inhibition of NO synthesis with $\mathrm{N}^{\mathbf{w}}$-nitro-L-arginine methyl ester (L-NAME), or (c) inhibition of cyclooxygenase with indomethacin. Both endothelial disruption and L-NAME augmented pressure-induced constriction in free-flow but not no-flow Af-Arts, abolishing the differences between the two. However, indomethacin had no effect in either free-flow or no-flow Af-Arts. These results suggest that intraluminal flow attenuates pressure-induced constriction in Af-Arts via endothelium-derived NO. Thus flow-stimulated NO release may be important in the fine control of glomerular hemodynamics. (J. Clin. Invest. 1995. 95:2741-2748.) Key words: myogenic responses • flow-induced vasodilation - endothelium-derived relaxing factor • glomerular hemodynamics • renal microvasculature
\end{abstract}

Address correspondence to Luis A. Juncos, M.D., Hypertension and Vascular Research Division, Henry Ford Hospital, 2799 West Grand Boulevard, Detroit, MI 48202. Phone: 313-876-2010; FAX: 313-8761479.

Received for publication 14 June 1994 and in revised form 24 January 1995.

J. Clin. Invest.

(c) The American Society for Clinical Investigation, Inc. 0021-9738/95/06/2741/08 \$2.00

Volume 95, June 1995, 2741-2748

\section{Introduction}

Myogenic response refers to the ability of vascular smooth muscle to constrict in response to increases in transmural pressure and dilate when the pressure is decreased. This response is important in establishing vascular tone and is thought to be closely linked to the regulation of regional blood flow and capillary pressure (1). In the kidney, increasing perfusion pressure causes myogenic constriction of the preglomerular resistance vessels (2-4), which (together with tubuloglomerular feedback) is thought to be important in the autoregulation of renal blood flow (RBF) ${ }^{1}$ and the glomerular filtration rate (GFR) (3-6). Among various preglomerular vascular segments, the glomerular afferent arteriole (Af-Art) is thought to play a major role in autoregulation.

The myogenic response may be modulated by neural, hormonal and metabolic factors, as well as physical factors such as luminal flow (7-12). In conduit arteries, flow is known to cause vasodilation (13-16), and it has been suggested that such dilation is mediated by nitric oxide (NO) released by the endothelium $(7,9,10,17)$. Indeed, shear stress associated with flow has been shown to be a major stimulus for endothelial release of NO $(14,18-20)$ and/or prostaglandins (PGs) (18, 21-23). On the other hand, microvascular responses to flow are remarkably variable and site specific (24-26). Thus it remains unclear whether flow modulates the myogenic response of the Af-Art, nor do we fully understand the role of the endothelium, NO or PGs in the myogenic response of the Af-Art, a crucial vascular segment to the control of glomerular hemodynamics.

The goals of the present study were to determine $(a)$ whether pressure-induced constriction of the Af-Art is modulated by flow; $(b)$ whether an intact endothelium is needed in order for flow to modulate pressure-induced constriction; and (c) whether NO and/or PGs are involved in the modulation. To examine these questions directly, we developed a novel in vitro preparation in which two Af-Arts are microperfused simultaneously, one with flow and the other without, and studied pressure-induced constriction before and after endothelial disruption or blockade of either NO or PG synthesis.

\section{Methods}

Isolation and microperfusion of the renal arterioles. We used a modification of a previously described method to isolate and microperfuse the renal arterioles $(27,28)$. Briefly, young male New Zealand white rabbits (1.5-2.0 kg), fed standard rabbit chow (Ralston Purina; St. Louis, MO) and tap water ad libitum, were anesthetized with intravenous sodium pentobarbital $(40 \mathrm{mg} / \mathrm{kg}$ ) and given an intravenous injection of heparin

1. Abbreviations used in this paper: Af-Art, afferent arteriole; Ef-Art, efferent arteriole; GFR, glomerular filtration rate; NO, nitric oxide; PG, prostaglandin; RBF, renal blood flow. 

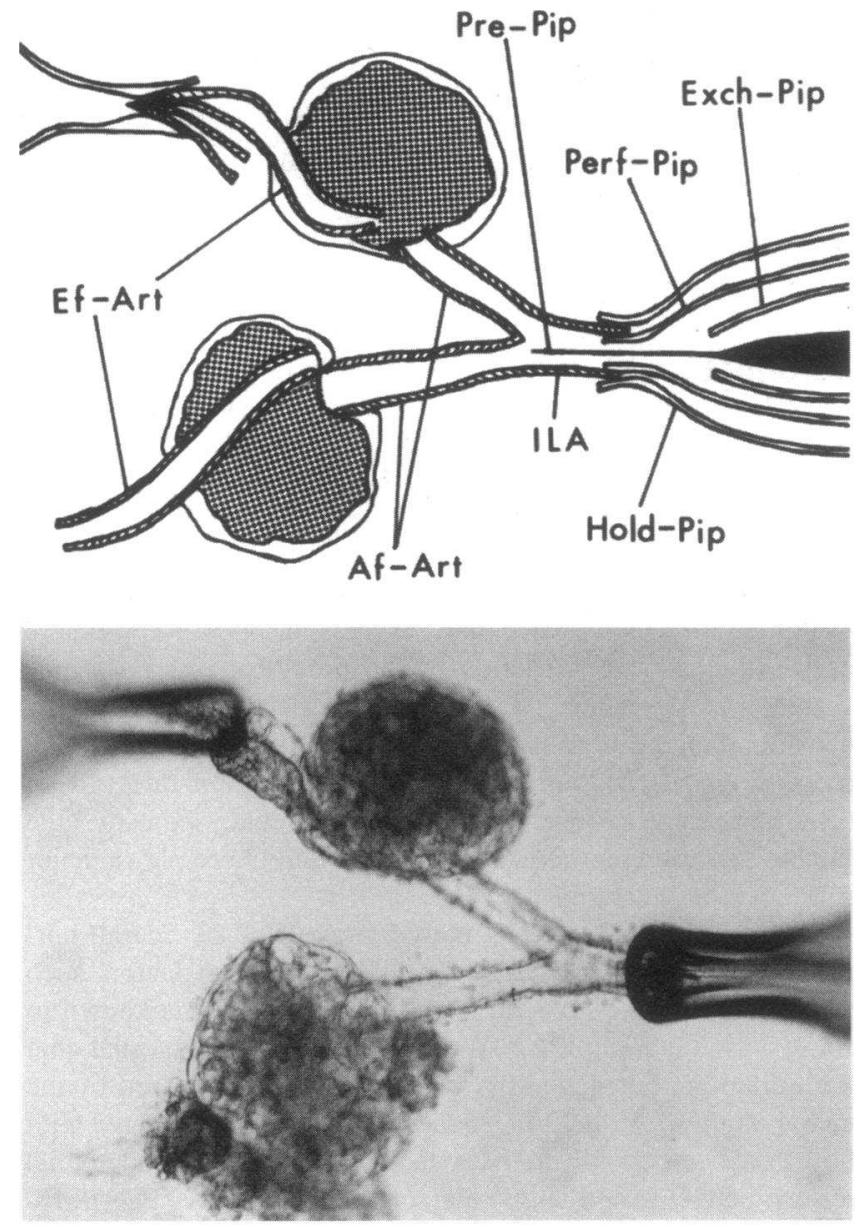

Figure 1. Schematic representation (top) and photograph (bottom) of the double Af-Art preparation. Hold-Pip, holding pipette; Perf-Pip, perfusion pipette; Exch-Pip, exchange pipette; Pre-Pip, pressure pipette; $A f-A r t$, afferent arteriole; $E f-A r t$, efferent arteriole; $I L A$, interlobular artery. Note: Since the arteriolar perfusate contained 5\% albumin, oncotic pressure built up in the glomerulus with the occluded Ef-Art and opposed the force of filtration, resulting in little or no flow through the corresponding Af-Art. Perfusion pressure was measured at the bifurcation of the interlobular artery using Landis' technique.

(500 U). The kidneys were removed and sliced along the corticomedullary axis. Slices were placed in ice-cold minimum essential medium (MEM; GIBCO BRL, Gaithersburg, MD) containing 5\% BSA (Sigma Chemical Co., St. Louis, MO). Using a stereomicroscope (SZH; Olympus, Tokyo, Japan), we microdissected a single sample consisting of the terminal segment of an interlobular artery together with two AfArts and their corresponding glomeruli and efferent arterioles (Ef-Art). The sample was transferred to a temperature-regulated chamber mounted on an inverted microscope (Diaphot; Nikon) and the Af-Arts perfused from the distal end of the interlobular artery. Fig. 1 shows a schematic representation (upper panel) and photograph (lower panel) of our preparation. The interlobular artery was drawn into the holding pipette, after which the perfusion pipette was advanced into the lumen of the interlobular artery and perfusion was begun. A fine pipette (pressure pipette) was advanced through the perfusion pipette into the interlobular artery to the point where it bifurcates into the two Af-Arts. Intraluminal pressure at this point was measured with the pressure pipette, using Landis' technique. The arterioles were perfused with oxygenated medium 199 containing 5\% BSA (M199-5\%BSA), and perfusion (intraluminal) pressure was maintained at $60 \mathrm{mmHg}$ throughout the equilibra- tion period. The driving force of the perfusate was provided by a pressurized tank of air attached to the proximal end of the perfusion pipette. An air regulator positioned between the tank and the perfusion pipette was manipulated to control intraluminal pressure as measured with the pressure pipette.

The bath, which was exchanged continuously, was identical to the arteriolar perfusate except that it contained $0.1 \%$ (instead of 5\%) BSA. Microdissection and cannulation of the arteriole were completed within $90 \mathrm{~min}$ at $8^{\circ} \mathrm{C}$, after which the bath was gradually warmed to $37^{\circ} \mathrm{C}$ for the rest of the experiment. Once the temperature had stabilized, a 30min equilibration period was allowed. We then occluded one Ef-Art by sucking it into a holding pipette in a bent position (Fig. 1) and waited an additional $15 \mathrm{~min}$ before taking any measurements. Because the perfusate contained 5\% BSA, oncotic pressure increased in the glomerulus with the occluded Ef-Art, causing ultrafiltration to decrease: consequently, there was little or no flow through the corresponding Af-Art. Thus this preparation allowed us to observe two Af-Arts simultaneously, one with free-flowing perfusate (free-flow Af-Art) and the other with little or no flow (no-flow Af-Art). Images of the Af-Arts were displayed at magnifications up to $1,980 \times$ and recorded with a video system consisting of a camera (NC-70; DAGE-MTI), monitor (HR100; DAGEMTI) and video recorder (SLV-R5UC; Sony). Since pressure-induced constriction was strongest at the proximal segment (close to the interlobular artery), we measured the luminal diameter at this point using an image analysis system (Fryer, Carpentersville, IL).

To determine how effectively flow was reduced by occluding the Ef-Art, a single Af-Art with its corresponding glomerulus and Ef-Art was perfused with M199-5\% BSA containing free fluorescein isothiocyanate (FITC) (Sigma Chemical Co.). Changes in perfusate flow rates induced by occlusion of the Ef-Art were calculated using the concentration of FITC in the bath. Occluding the Ef-Art decreased the rate of flow by $89.9 \pm 0.3$ and $96.7 \pm 1.3 \%(n=4)$ when intra-arteriolar pressure was kept at 30 and $120 \mathrm{mmHg}$, respectively, thereby demonstrating the effectiveness of our method.

\section{Experimental protocols}

Time course of myogenic responses. To examine the time course of the myogenic response in (free-flow and no-flow) Af-Arts $(n=8)$, we monitored luminal diameter of Af-Arts for ten minutes while raising intraluminal pressure abruptly from 40 to $120 \mathrm{mmHg}$. We found that increasing intraluminal pressure caused a biphasic response, consisting of an initial constriction followed by partial return toward the basal diameter. Since the diameter stabilized completely within $5 \mathrm{~min}$, in the remaining experiments we measured the diameter 5 min after changing the pressure.

Pressure-response curves in free-flow and no-flow Af-Arts. To examine whether pressure-induced constriction is modulated by flow, the relationship between intraluminal pressure and luminal diameter was determined in free-flow and no-flow Af-Arts $(n=30)$. After the equilibration period, intraluminal pressure was decreased to $30 \mathrm{mmHg}$ (the diameter at this pressure was considered the basal diameter during the remainder of the pressure-response curve) and then increased in 30$\mathrm{mmHg}$ increments to a maximum of $120 \mathrm{mmHg}$. Pressure was maintained for $5 \mathrm{~min}$ at each step to ensure that the Af-Arts reached a steadystate diameter.

Upon completion of the initial pressure-response curve, pressure was decreased to $60 \mathrm{mmHg}$ and the occluded Ef-Art was released, allowing flow through the Af-Art to resume. The Af-Arts were then treated according to one of the five protocols described below, after which the Ef-Art was re-occluded. Following an additional 15-minute equilibration period, a second pressure-response curve was obtained.

Time controls. Five of the above preparations were utilized as time controls. After completion of the initial pressure-response curve, the Af-Arts were perfused with the control medium for $30 \mathrm{~min}$, the Ef-Art re-occluded and a second pressure-response curve obtained.

Passive pressure-diameter relationships. We determined pressurediameter relationships in Af-Arts which had been rendered passive ( $n$ $=6$ ). After the first pressure-response curve, $10^{-4} \mathrm{M}$ sodium nitroprus- 


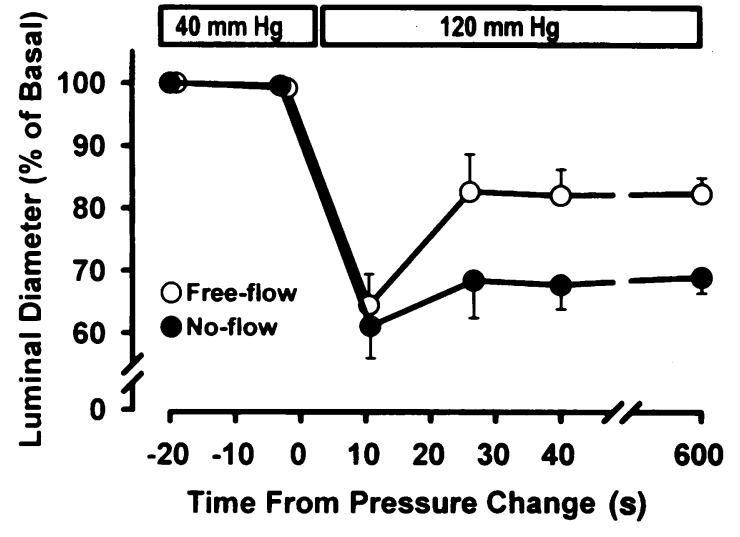

Figure 2. Characterization and time course of myogenic responses in free-flow and no-flow Af-Arts $(n=8)$. Note: Pressure was increased abruptly from 40 to $120 \mathrm{mmHg}$ and maintained at this level for $10 \mathrm{~min}$.

side was added to both the bath and arteriolar perfusate and maintained throughout the second pressure-response curve. At the end of the experiments, we confirmed that the Af-Arts did not constrict in response to $10^{-6} \mathrm{M}$ norepinephrine.

Effect of endothelial disruption. We tested whether the endothelium plays a role in flow modulation of the myogenic response. After the first pressure-response curve was obtained, seven preparations were perfused for $10 \mathrm{~min}$ with M199-5\% BSA containing anti-human factor VIII-related antigen antibody $(14.29 \mu \mathrm{g} / \mathrm{ml}$; Atlantic Antibodies $)$ and $2 \%$ guinea pig complement (Sigma Chemical Co.). This was followed by a 20-min washout period during which the arterioles were perfused with M199-5\% BSA containing neither antibody nor complement. The Ef-Art was then re-occluded and the pressure-response curve repeated. We have previously demonstrated that the antibody-complement treatment selectively disrupts endothelial cells without altering vascular smooth muscle cells (29). At the end of each experiment, we confirmed that the Af-Arts did not dilate in response to $10^{-5} \mathrm{M}$ acetylcholine, an endothelium-dependent vasodilator.

Effect of NO synthesis inhibition. We examined whether NO plays a role in flow modulation of the myogenic response. Following the first pressure-response curve, $10^{-4} \mathrm{M}$ nitro-L-arginine methyl ester (LNAME), an NO synthesis inhibitor, was added to the arteriolar perfusate and maintained throughout the second pressure-response curve $(n=7)$. We have previously demonstrated that this concentration of L-NAME abolishes acetylcholine-induced vasodilation in isolated perfused AfArts $(27,30)$.

Effect of cyclooxygenase inhibition. To examine whether PGs are involved in the flow modulation of pressure-induced constriction, we added $5 \times 10^{-5} \mathrm{M}$ indomethacin (Sigma Chemical Co.), a cyclooxygenase inhibitor, to both the bath and perfusate after the initial pressureresponse curve $(n=5)$. We have previously shown that this dose of indomethacin blocks the effect of $10^{-4} \mathrm{M}$ arachidonic acid on renin release and augments angiotensin II-induced constriction in isolated rabbit Af-Arts $(31,32)$.

\section{Data analysis}

Data are expressed as mean \pm SEM. Paired $t$ tests were used to examine whether the diameter at a given pressure differed from the baseline value (at $30 \mathrm{mmHg}$ ) within each group. Univariate repeated-measures ANOVA with the Greenhouse-Geisser sphericity correction was used to test whether the groups (free-flow vs. no-flow, or before vs. after treatment) differed with respect to the rate of change across the various periods. For this analysis, $P<0.05$ was considered significant. Paired $t$ tests were used to examine whether the change in diameter at a given pressure differed before and after treatment. When more than one measurement was made, Bonferroni's multiple comparison adjustment was used to reduce the significance level.

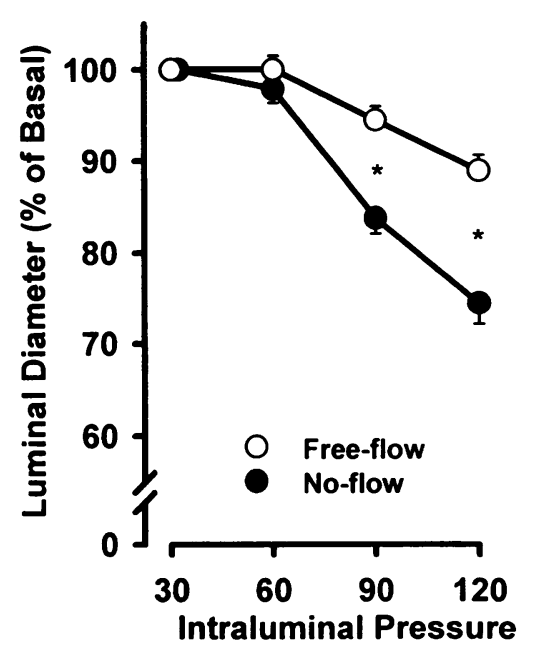

Figure 3. Pressure-diameter relationships in freeflow and no-flow Af-Arts $(n=30 ; * P<0.001$ for free-flow vs. no-flow Af-Arts ). Note that pressure-induced constriction was significantly stronger in no-flow AfArts.

\section{Results}

Time course of myogenic responses. At $40 \mathrm{mmHg}$, luminal diameter of free-flow and no-flow Af-Arts was 17.8 \pm 0.7 and $18.2 \pm 0.4 \mu \mathrm{m}$, respectively $(n=16)$. Increasing the pressure abruptly to $120 \mathrm{~mm} \mathrm{Hg}$ caused a biphasic constriction in both free-flow and no-flow Af-Arts (Fig. 2). Almost invariably, the segment which constricted the most was the proximal portion, just distal from the bifurcation of the interlobular artery; thus measurements were made at this point. The initial constriction, which occurred within $10 \mathrm{~s}$ after increasing the pressure, was of similar magnitude in both free-flow and no-flow Af-Arts; luminal diameter decreased to $64.5 \pm 5.0$ and $61.1 \pm 5.1 \%$ of 40 $\mathrm{mmHg}$ levels, respectively. This initial constriction was followed by partial relaxation over the next $30 \mathrm{~s}$ which was greater in free-flow Af-Arts; the diameter increased to $82.7 \pm 2.4$ and $68.5 \pm 2.5 \%$ of baseline in free-flow and no-flow Af-Arts, respectively $(P<0.005$ for free-flow vs. no-flow Af-Arts $)$, suggesting that flow modulates the late, or static component of pressure-induced constriction. Small oscillations in diameter continued for 2-3 min, after which the diameter stabilized.

Pressure-response curves in free-flow and no-flow Af-Arts. At $30 \mathrm{~mm} \mathrm{Hg}$, the mean luminal diameter of free-flow and noflow Af-Arts was 17.6 \pm 0.4 and $18.3 \pm 0.4 \mu \mathrm{m}$, respectively ( $n$ $=30$ ). Both constricted in response to increased pressure (Fig. 3); however, constriction was significantly stronger in no-flow Af-Arts. At 90 and $120 \mathrm{mmHg}$, the diameter of no-flow Af-Arts decreased to $83.8 \pm 1.7$ and $74.4 \pm 2.3 \%$ of baseline, respectively, whereas the corresponding values for free-flow Af-Arts were $94.5 \pm 1.4$ and $88.9 \pm 1.7 \%$.

Time controls. Luminal diameter of free-flow Af-Arts at 30, 60,90 , and $120 \mathrm{mmHg}$ was $18.8 \pm 0.7,19.3 \pm 0.9,18.2 \pm 1.2$, and $16.7 \pm 1.1 \mu \mathrm{m}$, respectively, for the first pressure-response curve $(n=5)$. The second curve was similar to the first one, with corresponding values of $19.4 \pm 0.7,19.6 \pm 0.7,18.2 \pm 1.0$, and $17.2 \pm 1.1 \mu \mathrm{m}$. Likewise, there was no difference between the two curves in no-flow Af-Arts: luminal diameter at 30,60 , 90 , and $120 \mathrm{mmHg}$ was $20.8 \pm 1.1,19.1 \pm 0.8,17.1 \pm 0.8$, and $15.3 \pm 0.6 \mu \mathrm{m}$, respectively, for the first curve and 19.2 \pm 0.9 , $18.7 \pm 0.7,16.9 \pm 0.6$, and $15.8 \pm 0.4 \mu \mathrm{m}$ for the second.

Passive pressure-diameter relationships. Sodium nitroprusside $\left(10^{-4} \mathrm{M}\right)$ did not alter luminal diameter of either freeflow or no-flow Af-Arts at $30 \mathrm{mmHg}$. Increasing intraluminal 

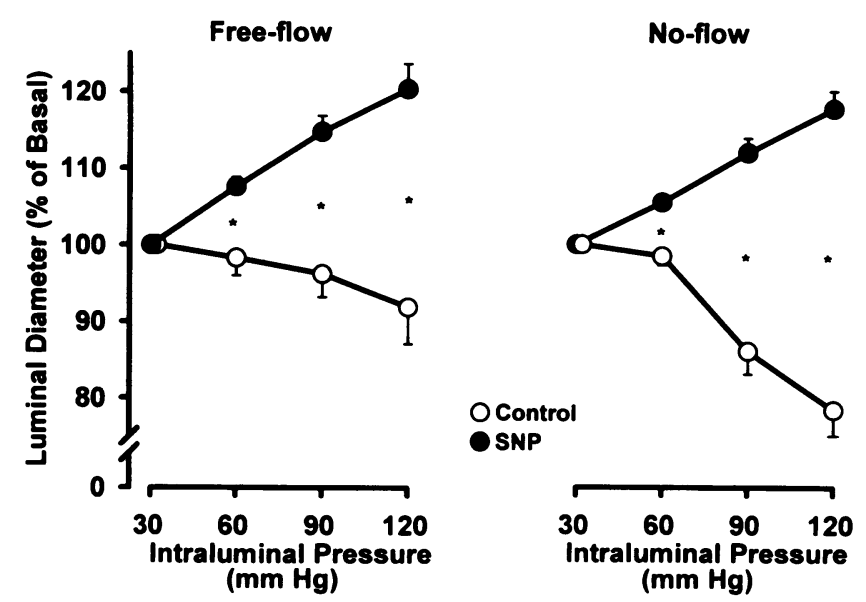

Figure 4. Pressure-diameter relationships in free-flow (left) and no-flow Af-Arts (right) in the absence and presence of sodium nitroprusside ( $n$ $=6$; $P<0.005$ for control vs. sodium nitroprusside-treated Af-

Arts). Note that with sodium nitroprusside, vessels dilated passively in response to increases in intraluminal pressure. Increases in pressure produced almost identical increases in diameter in free-flow and noflow Af-Arts after sodium nitroprusside.

pressure in the presence of sodium nitroprusside elicited a continuous increase in the diameter throughout the pressure range tested. This increase was almost identical in free-flow and noflow Af-Arts: diameter increased to $107.6 \pm 1.2,114.7 \pm 2.2$, and $120.3 \pm 3.3 \%$ of baseline in free-flow Af-Arts and to $105.5 \pm 0.9$, $111.9 \pm 1.9$, and $117.6 \pm 2.3 \%$ in no-flow Af-Arts at 60,90 , and $120 \mathrm{~mm} \mathrm{Hg}$, respectively ( $n=6$; Fig. 4).

Effect of endothelial disruption. Treatment with anti-factor VIII-related antigen antibody and complement did not alter basal luminal diameter in either free-flow or no-flow Af-Arts; the diameter of free-flow Af-arts before and after the treatment was $17.4 \pm 0.9$ and $17.2 \pm 0.6 \mu \mathrm{m}$, respectively, while that of noflow Af-Arts was $18.1 \pm 0.5$ and $18.0 \pm 0.5 \mu \mathrm{m}(n=7)$. Fig. 5
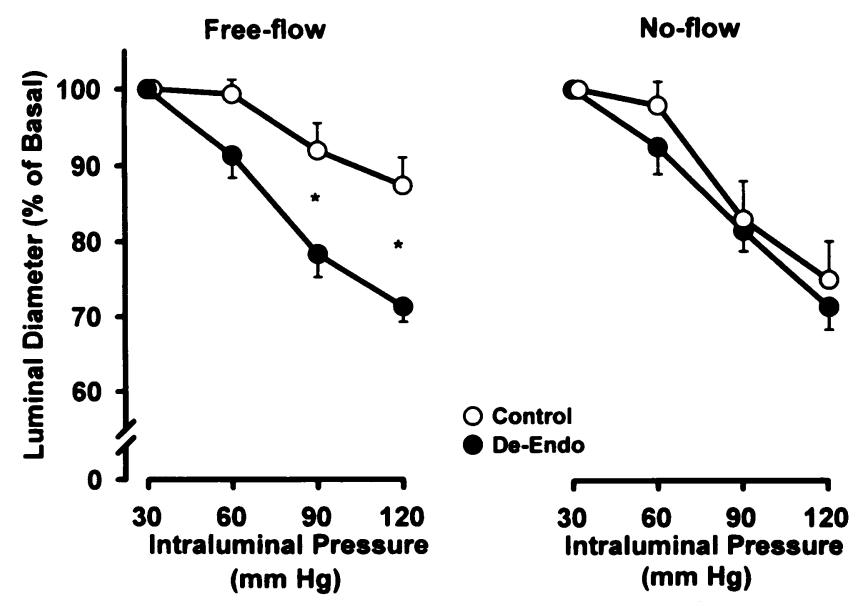

Figure 5. Pressure-diameter relationships in free-flow (left) and no-flow Af-Arts (right) before and after endothelial disruption (De-endo); $n$ $=7 ; * P<0.01$ for control vs. de-endo Af-Arts. Note that endothelial disruption only augmented pressure-induced constriction in free-flow Af-Arts, thus eliminating the difference between free-flow and no-flow Af-Arts.

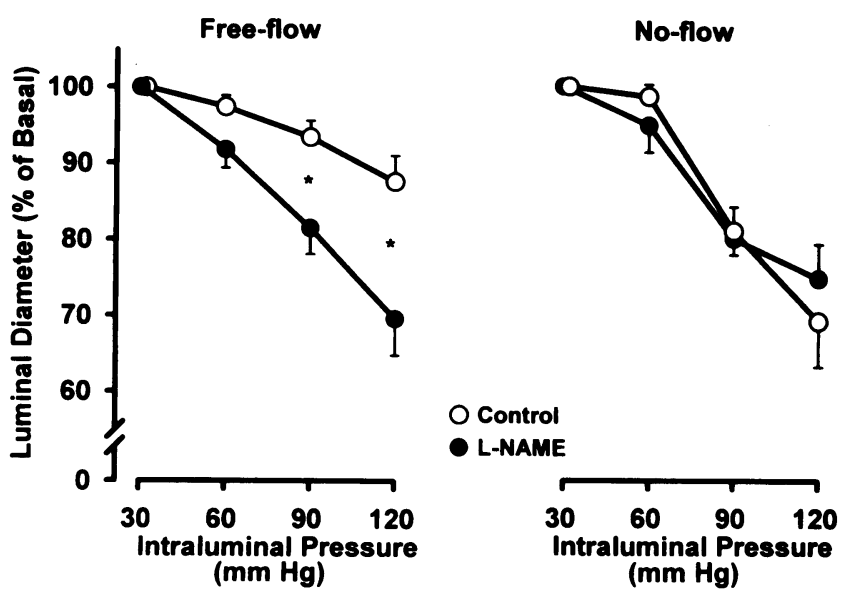

Figure 6. Pressure-diameter relationships in free-flow (left) and no-flow Af-Arts (right) in the absence and presence of L-NAME $(n=7 ; * P$ $<0.01$ for control vs. L-NAME-treated Af-Arts). Note that L-NAME augmented pressure-induced constriction in free-flow but not no-flow Af-Arts.

depicts the myogenic response before and after the antibodycomplement treatment. In free-flow Af-Arts, the treatment significantly augmented pressure-induced constriction, with diameter decreasing to $78.3 \pm 3.9$ and $71.3 \pm 2.0 \%$ of baseline at 90 and $120 \mathrm{mmHg}$, respectively, compared with $91.9 \pm 3.5$ and $87.6 \pm 3.7 \%$ before the treatment. In marked contrast, pressureinduced constriction remained unchanged in no-flow Af-Arts. Consequently, after the antibody-complement treatment, pressure-induced constriction was no longer different in free-flow and no-flow Af-Arts.

Effect of NO synthesis inhibition. After L-NAME treatment, luminal diameter of free-flow and no-flow Af-Arts decreased significantly by $12.8 \pm 2.6 \%$ (from $18.1 \pm 1.2$ to $15.8 \pm 1.3 \mu \mathrm{m}$ ) and $12.0 \pm 2.6 \%$ (from $17.8 \pm 1.4$ to $15.3 \pm 1.1 \mu \mathrm{m}$ ), respectively $(n=7)$. L-NAME augmented pressure-induced constriction in free-flow but not no-flow Af-Arts (Fig. 6). Thus L-NAME also completely abolished the difference in pressure-induced constriction between free-flow and no-flow Af-Arts.

Effect of cyclooxygenase inhibition. Indomethacin treatment did not alter basal luminal diameter of either free-flow or noflow Af-Arts; the diameter of free-flow Af-Arts before and after indomethacin was $15.7 \pm 0.8$ and $16.7 \pm 0.5 \mu \mathrm{m}$, respectively, while that of no-flow Af-Arts was 17.2 \pm 0.8 and 17.3 $\pm 0.8 \mu \mathrm{m}$, respectively $(n=5)$. Indomethacin did not alter pressureinduced constriction in either free-flow or no-flow Af-Arts (Fig. 7).

\section{Discussion}

In the present study, we examined the Af-Art response to increases in intraluminal pressure in the presence and absence of intraluminal flow, as well as the pathways through which flow exerts its effects. Our findings provide the first direct evidence that pressure-induced constriction in Af-Arts is modulated by luminal flow. We found that the effect of flow on pressureinduced constriction is dependent on an intact endothelium. Furthermore, our results suggest that the endothelium-derived factor responsible for the flow effect may be NO rather than prostanoids. 


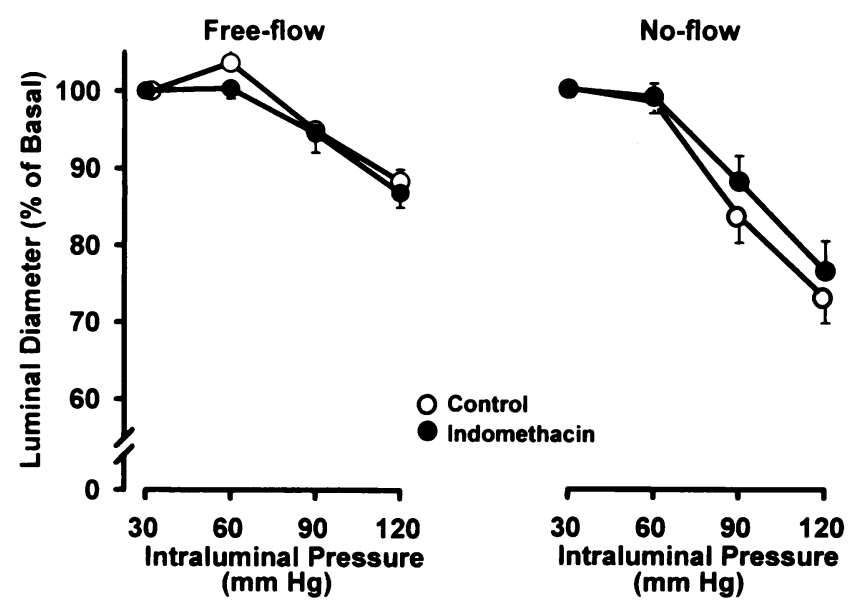

Figure 7. Pressure-diameter relationships in free-flow (left) and no-flow Af-Arts (right) in the absence and presence of indomethacin $(n=5)$. Note that indomethacin had no effect on pressure-induced constriction in either free-flow or no-flow Af-Arts.

We first characterized myogenic responses of Af-Arts to abrupt increases in pressure. We found that both free- and noflow Af-Arts responded in a biphasic manner, with an initial constriction followed by a partial return toward baseline. The initial constriction was no different in free-flow and no-flow Af-Arts, whereas relaxation during the second phase was greater in free-flow Af-Arts. These findings suggest that flow only modulates the late component of myogenic constriction. It may be that since the decrease in luminal diameter induced by the myogenic response would increase shear stress on the endothelium much more in the presence rather than the absence of flow, the release of NO and hence relaxation would be greater in freeflow Af-Arts. However, it is interesting to note that even in noflow Af-Arts, the sustained response remained less than the transient response (though the difference was small). Such partial relaxation under no-flow conditions was also observed in arterioles from the other vascular beds $(33,34)$. As discussed previously (34), the nature of this biphasic myogenic response to an abrupt increase in intravascular pressure may be related to the presence of rate-sensitive and static-sensitive components of myogenic responses.

We found that Af-Arts constricted significantly in response to increased luminal pressure, especially under no-flow conditions; the magnitude of actual development of vascular tone becomes apparent when one compares diameters before and after the Af-Arts were rendered passive with sodium nitroprusside. In contrast, previous studies showed only weak myogenic constriction in isolated rat and rabbit Af-Arts even in no-flow conditions $(35,36)$. This discrepancy may be related to the sites where the myogenic responses were measured. In the present study, we took our measurements at the proximal segment of the Af-Art (close to the interlobular artery), since this segment almost always exhibited distinctly stronger constriction than more distal segments. However, when single Af-Arts were used, the diameter was measured at more distal segments because the proximal segments were used for cannulation and therefore could not be observed. Alternatively, the discrepancy could be due to the different conditions and techniques used to isolate and cannulate the arterioles. Indeed, the myogenic response in isolated vessels is quite susceptible to trauma and is often abol- ished when microperfusion and cannulation procedures are used (37-39). Thus we took extreme precautions during preparation. In particular, we chose to occlude the Ef-Art to eliminate luminal flow through the Af-Art, since this procedure does not cause stretching of the Af-Art. Furthermore, since occluding and releasing the Ef-Art do not affect the Af-Art, we could obtain pressure-response curves before and after treating the Af-Arts.

The dominant response to flow in large conduit arteries of various vascular beds, including the femoral, brachial, cerebral, pulmonary, and coronary circulations, is vasodilation (13-16, $24,25)$. Recently this phenomenon has also been demonstrated at the arteriolar level of the mesenteric (40), skeletal muscle $(41,42)$, and coronary $(7,8,17,26)$ circulations. In the present study, we observed that pressure-induced constriction was significantly weaker in free-flow than no-flow Af-Arts. It may be argued that the stronger constriction observed in no-flow AfArts could be due to higher pressure at the site of measurement, since pressure would drop along free-flow Af-Arts more steeply than no-flow Af-Arts. However, we consider this unlikely, since all measurements were made in the proximal segment of the Af-Art (within $50 \mu \mathrm{m}$ from the bifurcation) and the length of Af-Arts exceeded $200 \mu \mathrm{m}$ in most cases. Nevertheless, we examined the pressure-diameter relationship in both free-flow and no-flow Af-arts before and after treatment with sodium nitroprusside, finding that it rendered Af-Arts completely passive and luminal diameter increased linearly as intraluminal pressure was elevated. Importantly, the increase in diameter was virtually identical, suggesting that intraluminal pressure at the site of measurement did not differ significantly between free-flow and no-flow Af-Arts. Moreover, if the difference in pressure-induced constriction was simply due to different pressure, it should not be abolished by endothelial disruption or LNAME.

The present study showed that endothelial disruption augmented pressure-induced constriction only in free-flow but not in no-flow Af-Arts, thereby eliminating the difference between the two. This suggests that flow modulation of the myogenic response is endothelium-dependent in the Af-Art. Since flow is known to stimulate the release of both NO $(14,18-20)$ and $\mathrm{PGI}_{2}(18,21-23)$ from endothelial cells, we examined their role using L-NAME and indomethacin, respectively. Like endothelial disruption, L-NAME was found to abolish the difference between free-flow and no-flow Af-Arts whereas indomethacin had no effect, suggesting that NO rather than PGs is involved in flow modulation of the myogenic response in Af-Arts. Although indomethacin had no effect under our experimental conditions, we do not consider Af-Arts to produce an insignificant amount of PGs; nor is it likely that the lack of modulation by indomethacin is due to insufficient blockade of cyclooxygenase, since we have shown that the same dose augments Ang II-induced constriction in isolated microperfused rabbit Af-Arts (32). Rather, we speculate that endogenous PGs do not affect myogenic responses, since we have experimental evidence that isolated Af-Arts do synthesize PGs, which in turn control both renin release and angiotensin II action $(32,43)$. Consistent with our observation, Rubanyi et al. (14) found that although flow increased release of both $\mathrm{NO}$ and 6-keto-prostaglandin $\mathrm{F}_{1 \mathrm{a}}$, only NO significantly counteracted myogenic tone in the femoral arteries. However, it appears that there is considerable heterogeneity among species and/or vascular beds, since some studies suggest that flow-induced vasodilation is mediated by $\mathrm{NO}(7,9$, $10,17)$, whereas others suggest involvement of cyclooxygenase 
products (41). In addition, endothelium-derived contracting factors (such as $\mathrm{PGH}_{2}$ and thromboxane $\mathrm{A}_{2}$ ) may be involved in vascular responses induced by flow under certain conditions. Thus the lack of potentiation by indomethacin in our study might be related in part to its pharmacological action, which blocks not only vasodilator PGs but also contracting factors.

Whether the endothelium mediates myogenic contractile responses remains a controversial issue (44). We found that disruption of the endothelium did not eliminate pressure-induced constriction but rather augmented it in the case of free-flow AfArts. Our findings are consistent with most other studies using small resistant arteries $(37,45-47)$, and suggest that myogenic contractile responses are not mediated by the endothelium. In contrast, Eskinder et al. (48) found that the myogenic response was attenuated when the endothelium was removed by air perfusion in canine small renal arteries (200-500 $\mu \mathrm{m}$ in diameter). However, the same group recently reported that removal of the endothelium by mechanical rubbing did not affect myogenic responses (49). They suggested that myogenic activation of small renal arteries is independent of the endothelium and that the method of endothelial removal may affect conclusions regarding the role of the endothelium in mediating the myogenic response.

It is noteworthy that L-NAME caused a significant decrease in the basal diameter of both free-flow and no-flow Af-Arts, whereas endothelial disruption with antibody/complement did not constrict either one. These results are consistent with those of Kuo et al. (7) who found that L-NAME, but not de-endothelialization, constricted pig coronary arterioles. The reason for this may be that endothelial disruption eliminated both dilating and constricting factors derived from the endothelium whereas LNAME only eliminated NO. It is also possible that L-NAMEinduced vasoconstriction is due to inhibition of NO synthesis by Af-Art vascular smooth muscle cells and/or some action(s) other than inhibition of NO (50-52). In view of our results suggesting that flow stimulates NO release, it is also interesting to note that L-NAME constricted both no-flow and free-flow Af-Arts to the same extent at a luminal pressure of $30 \mathrm{mmHg}$. While the reason for this is unclear, there might be basal release of NO independent of flow; thus L-NAME still caused constriction in the absence of flow. In addition, because flow did not seem to modulate vascular tone until the pressure reached 60 mmHg (Fig. 3), L-NAME would have little, if any, differential effect on free-flow and no-flow Af-Arts.

Consistent with our finding that L-NAME augmented myogenic responses in free-flow Af-Arts, a recent preliminary study by Hayashi et al. (53) reported that pressure-induced constriction of the Af-Art was augmented by an NO synthase inhibitor in isolated hydronephrotic kidneys of spontaneously hypertensive and normotensive Wistar-Kyoto rats. In contrast, Imig et al. (54) reported that when juxtamedullary nephrons were perfused with a cell-free solution in vitro, inhibition of NO synthesis had little effect on changes in Af-Art diameter induced by increasing perfusion pressure, even though it significantly decreased basal diameter (at a renal perfusion pressure of $80 \mathrm{mmHg}$ ). While the reason for these discrepancies is not clear, they may be related to differences in species/strains, nephron populations studied (superficial vs. juxtamedullary) and/or preparations used. The presence (Imig's preparation) or absence of tubular function (Hayashi's and our preparations) may be particularly important. It may be that in the juxtamedullary nephrons, NO inhibition could alter glomerular-tubular balances and/or in- crease sodium reabsorption by nephron segments proximal to the macula densa, leaving less sodium chloride free to reach the macula densa and hence a lesser contribution of tubuloglomerular feedback to the Af-Art constriction induced by stepwise increases in renal perfusion pressure. It should also be noted that although renal perfusion pressure was kept constant in Imig's and Hayashi's studies, pressure in the Af-Art may not have been the same in the presence and absence of NO synthase inhibition, since it significantly augmented pressure-induced constriction in the interlobular artery, which is immediately upstream from the Af-Art.

Our study demonstrates the existence of dynamic interactions between pressure and flow in the control of glomerular hemodynamics, which may have important physiological and pathological implications. It is well known that when renal perfusion pressure is increased above the autoregulatory threshold, renal vascular resistance increases just enough to precisely maintain a constant RBF and GFR. Such constriction (decrease in luminal diameter) in the face of constant luminal flow would increase shear stress on the endothelium, resulting in enhanced production and release of NO which in turn may participate in fine tuning of vascular resistance. Since myogenic constriction of the Af-Art contributes significantly to autoregulation (2-6), our results may predict that inhibition of NO synthesis would decrease RBF and GFR to a greater extent when renal perfusion is high; in other words, increasing perfusion pressure would actually decrease RBF and GFR (superautoregulation). Although some studies suggest this is the case (55), most others have found that autoregulation is well maintained (but at a lower RBF) during NO synthesis inhibition (56-58). While the reason for this discrepancy is unknown, other compensatory mechanisms may be capable of maintaining RBF in vivo in normal animals. Since macula densa-mediated tubuloglomerular feedback is another major mechanism involved in autoregulation, it is tempting to speculate that tubuloglomerular feedback is modulated to compensate for enhanced myogenic response. However, we (59) and others (60) have shown that inhibition of NO synthase in the macula densa actually augments the AfArt constriction induced by high $\mathrm{NaCl}$ at the macula densa. Nevertheless, it is possible that in intact animals, due to possible modulation of the glomerular-tubular balance and increased sodium reabsorption by tubular segments proximal to the macula densa, sodium chloride concentration at the macula densa may fall significantly, so that tubuloglomerular feedback contributes less to renal autoregulation. On the other hand, the importance of flow- or shear stress-associated NO release may become more apparent in pathological conditions such as polycythemia rubra vera. In patients with polycythemia, RBF and GFR are often within the normal range despite high blood viscosity (61, 62 ), which, if left unopposed, results in increased vascular resistance (63). Since heightened blood viscosity increases shear stress on the endothelium, NO may play an important role in the maintenance of RBF and GFR. Indeed, Wilcox et al. (64) recently reported that $\mathrm{L}-\mathrm{NAME}$-induced systemic and renal vasoconstriction was greatly augmented in rats with erythropoietin-induced polycythemia. Further studies are needed in order to clarify the interaction between pressure and flow as well as the possible role of NO in various physiological and pathological conditions where autoregulation is altered.

In summary, in order to study the influence of luminal flow on the myogenic response of the Af-Art, we have developed a novel in vitro preparation in which two Af-Arts are perfused 
simultaneously, one with luminal flow and the other without. Our results demonstrate that the myogenic response is significantly attenuated by the presence of luminal flow and that this modulation is dependent on an intact endothelium and NO synthesis. Thus flow-associated NO production (and release) by the endothelium may play an important role in the control of glomerular hemodynamics.

\section{Acknowledgments}

This study was supported by National Institutes of Health (NIH) grants HL-46518 and HL-28982. S. Ito is the recipient of an Established Investigatorship Award of the American Heart Association and J. Garvin is the recipient of a Research Career Developmental Award of NIH.

\section{References}

1. Johnson, P. C. 1980. The myogenic response. In Handbook of Physiology. The Cardiovascular System. Vascular Smooth Muscle. Sect. 2, vol. II, chapt. 15. Am. Physiol. Soc. Bethesda, MD. 409-442.

2. Robertson, C. R., W. M. Deen, J. Troy, and B. M. Brenner. 1972. Dynamics of glomerular filtration in the rat. III. Hemodynamics and autoregulation. Am. J. Physiol. 223:1191-1200.

3. Carmines, P., E. Inscho, J. Ortenberg, and A. Cook. 1991. Determinants of renal microvascular autoregulatory behavior in normal and hypertensive rats. Kidney Int. 39 [ suppl 32]:1402-1408.

4. Moore, L., and D. Casellas. 1990. Tubuloglomerular feedback dependence of autoregulation in rat juxtamedullary afferent arterioles. Kidney Int. 37:14021408.

5. Navar, L. G. 1978. Renal autoregulation: perspectives from whole kidney and single nephron studies. Am. J. Physiol. 234:F357-F370.

6. Aukland K., and A. Oien. 1987. Renal autoregulation: models combining tubuloglomerular feedback and myogenic responses. Am. J. Physiol. 252:F768F783.

7. Kuo, L., W. M. Chilian, and M. J. Davis. 1991. Interaction of pressureand flow-induced responses in porcine coronary resistance vessels. Am. J. Physiol. 261:H1706-H1715.

8. Kuo, L., F. Arko, W. M. Chilian, and M. J. Davis. 1993. Coronary venular responses to flow and pressure. Circ. Res. 72:607-615.

9. Griffith, T. M., and D. H. Edwards. 1990. Myogenic autoregulation of flow may be inversely related to endothelium-derived relaxing factor activity. Am. J. Physiol. 258:H1171-H1180.

10. Pohl, U., H. Klaus, A. Huang, and E. Bassenge. 1991. EDRF-mediated shear-induced dilation opposes myogenic vasoconstriction in small rabbit arteries. Am. J. Physiol. 261:H2016-H2023.

11. Faber, J., and G. Meininger. 1990. Selective interaction of $\alpha$-adrenoceptors with myogenic regulation of microvascular smooth muscle. Am. J. Physiol. 259:H1126-H1133.

12. Meininger, G., and J. Faber. 1991. Adrenergic facilitation of myogenic response in skeletal muscle arterioles. Am. J. Physiol. 260:H1424-H1432.

13. Hilton, S. 1959. Peripheral arterial conducting mechanism underlying dilatation of the femoral artery and concerned in functional vasodilation in skeletal muscle. J. Physiol. (Lond.). 149:93-97.

14. Rubanyi, G., J. C. Romero, and P. Vanhoutte. 1986. Flow-induced release of endothelium-derived relaxing factor. Am. J. Physiol. 250:H1145-H1 149.

15. Kaiser, L., S. Hull, and H. Sparks. 1986. Methylene blue and ETYA block flow-dependent dilation in canine femoral artery. Am. J. Physiol. 250:H974H981.

16. Pohl, U., J. Holtz, R. Busse, and E. Bassenge. 1986. Crucial role of the endothelium in the vasodilation response to increased flow in vivo. Hypertension. 8:37-44.

17. Kuo, L., W. Chilian, and M. Davis. 1990. Endothelium-dependent, flowinduced dilation of isolated coronary arterioles. Am. J. Physiol. 259:H1063H1070.

18. Nollert, M., S. Diamond, and L. McIntire. 1991. Hydrodynamic shear stress and mass transport modulation of endothelial cell metabolism. Biotechnol. Bioeng. 38:588-602.

19. Buga, G., M. Gold, J. Fukuto, and L. Ignarro. 1991. Shear stress-induced release of nitric oxide from endothelial cells grown on beads. Hypertension. 17:187-193.

20. Hutcheson, I., and T. Griffith. 1991. Release of endothelium-derived relaxing factor is modulated both by frequency and amplitude of pulsatile flow. Am. J. Physiol. 261:H257-H262.

21. van Grondelle, A., G. Worthen, D. Ellis, M. Mathias, R. Murphy, R. Streifl,
J. Reeves, and N. Voelkel. 1984. Altering hemodynamic variables influences $\mathrm{PGI}_{2}$ production by isolated lungs and endothelial cells. J. Appl. Physiol. 57:388-395.

22. Frangos, J., S. Eskin, L. McIntire, and C. Ives. 1985. Flow effects on prostacyclin production by cultured human endothelial cells. Science (Wash. DC). 227:1477-1479.

23. Bhagyalakshmi, A., and J. Frangos. 1989. Mechanism of shear-induced prostacyclin production in endothelial cells. Biochem. Biophys. Res. Commun. 158:31-37.

24. Garcia-Roldan, J. L., and J. Bevan. 1990. Flow-induced constriction and dilation of cerebral resistance arteries. Circ. Res. 66:1445-1448.

25. Bevan, J., and I. Laher. 1991. Pressure and flow-dependent vascular tone. FASEB (Fed. Am. Soc. Op. Biol.) J. 5:2267-2273.

26. Kuo, L., M. Davis, and W. Chilian. 1992. Endothelial modulation of arteriolar tone. News Physiol. Sci. 7:5-10.

27. Ito, S., C. S. Johnson, and O. A. Carretero. 1991. Modulation of angiotensin II-induced vasoconstriction by endothelium-derived relaxing factor in the isolated microperfused rabbit afferent arteriole. J. Clin. Invest. 87:1656-1663.

28. Ito, S., and O. A. Carretero. 1990. An in vitro approach to the study of macula densa-mediated glomerular hemodynamics. Kidney Int. 38:1206-1210.

29. Juncos, L. A., S. Ito, O. A. Carretero, and J. L. Garvin. 1994. Removal of endothelium-dependent relaxation by antibody and complement in afferent arterioles. Hypertension 23 [suppl I]:I-54-I-59.

30. Ito, S., S. Arima, Y. L. Ren, L. A. Juncos, and O. A. Carretero. 1993. Endothelium-derived relaxing factor/nitric oxide modulates angiotensin II action in the isolated microperfused rabbit afferent but not efferent arteriole. J. Clin. Invest. 91:2012-2019.

31. Itoh, S., and O. A. Carretero. 1985. Role of the macula densa in renin release. Hypertension. 7(Suppl. I):I49-I54.

32. Arima, S., Y. Ren, L. A. Juncos, O. A. Carretero, and S. Ito. 1994 Glomerular prostaglandins modulate vascular reactivity of the downstream efferent arterioles. Kidney Int. 45:650-658.

33. Sigurdsson, S., B. Johansson, and S. Mellander. 1977. Rate-dependent myogenic response of vascular smooth muscle during imposed changes in length and force. Acta Physiol. (Scand.). 99:183-189.

34. Davis, M., and P. Sikes. 1990. Myogenic responses of isolated arterioles: test for a rate-sensitive mechanism. Am. J. Physiol. 259:H1890-H1900.

35. Yuan, B., J. Robinette, and J. Conger. 1990. Effect of angiotensin II and norepinephrine on isolated rat afferent and efferent arterioles. Am. J. Physiol. 258:F741-F750.

36. Edwards, R. 1983. Segmental effects of norepinephrine and angiotensin II on isolated renal microvessels. Am. J. Physiol. 244:F526-F534.

37. Kuo, L., W. Chilian, and M. Davis. 1990. Coronary arteriolar myogenic response is independent of endothelium. Circ. Res. 66:860-866.

38. Duling, B., R. Gore, R. Dacey, and D. Damon. 1981. Methods for isolation, cannulation and in vitro study of single microvessels. Am. J. Physiol. 241:H108H116.

39. Dacey, R., and B. Duling. 1982. A study of rat intracerebral arterioles: methods, morphology, and reactivity. Am. J. Physiol. 243:H598-H606.

40. Smiesko, V., D. Lang, and P. Johnson. 1989. Dilator response of rat mesenteric arcading arterioles to increased blood flow velocity. Am. J. Physiol. 257:H1958-H1965.

41. Koller, A., and G. Kaley. 1990. Prostaglandins mediate arteriolar dilation to increased blood flow velocity in skeletal muscle microcirculation. Circ. Res. 67:529-534.

42. Koller, A., and G. Kaley. 1988. Endothelium regulates skeletal muscle microcirculation by a blood flow velocity sensing mechanism. Am. J. Physiol. 258:H916-H920.

43. Ito, S., O. A. Carretero, K. Abe, W. H. Beierwaltes, and K. Yoshinaga. 1989. Effect of prostanoids on renin release from rabbit afferent arterioles with and without macula densa. Kidney Int. 35:1138-1144.

44. Meininger, G. A., and M. J. Davis. 1992. Cellular mechanisms involved in the vascular myogenic response. Am. J. Physiol. 263:H647-H659.

45. Falcone, J., M. Davis, and G. Meininger. 1991. Endothelial independence of myogenic response in isolated skeletal muscle arterioles. Am. J. Physiol. 260:H130-H135.

46. Hwa, J. J., and J. A. Bevan. 1986. Stretch-dependent (myogenic) tone in rabbit ear resistant arteries. Am. J. Physiol. 250:H87-H95.

47. McCarron, J. G., G. Osol, and W. Halpern. 1989. Myogenic responses are independent of the endothelium in rat pressurized posterior cerebral arteries. Blood Vessels. 26:315-319.

48. Eskinder, H., D. Harder, and J. Lombard. 1990. Role of the vascular endothelium in regulating the response of small arteries of the dog kidney to transmural pressure elevation and reduced $\mathrm{PO}_{2}$. Circ. Res. 66:1427-1435.

49. Liu, Y., D. R. Harder, and J. H. Lombard. 1994. Myogenic activation of canine small renal arteries after nonchemical removal of the endothelium. Am. J. Physiol. 267:H302-H307.

50. Rosenblum, W., H. Nishimura, and G. Nelson. 1992. L-NMMA in brain microcirculation of mice is inhibited by blockade of cyclooxygenase and by superoxide dismutase. Am. J. Physiol. 262:H1343-H1349. 
51. Katusic, Z. 1991. Endothelium-independent contractions to $\mathrm{N}^{\mathrm{G}}$-monomethyl-L-arginine in canine basilar artery. Stroke. 22:1399-1404.

52. Thomas, G., and P. Ramwell. 1992. Interaction of non-arginine compounds with the endothelium-derived relaxing factor inhibitor, $\mathrm{N}^{\mathrm{G}}$-monomethyl-L-arginine. J. Pharmacol. Exp. Ther. 260:676-679.

53. Hayashi, K., H. Suzuki, and T. Saruta. 1994. Role of nitric oxide (NO) in the resetting of autoregulatory (myogenic) constriction of renal microvessels in SHR. J. Hypertens. 12(Suppl 3):S222 (abstract).

54. Imig, J. D., D. Gebremedhin, D. R. Harder, and R. J. Roman. 1993. Modulation of vascular tone in renal microcirculation by erythrocytes: role of EDRF. Am. J. Physiol. 264:H190-H195.

55. Salom, M. G., V. Lahera, F. J. Fenoy, R. Roman, and J. C. Romero. 1990. Role of endothelium-derived relaxing factor (EDRF) in the renal response to changes in renal perfusion pressure. J. Am. Soc. Nephrol. 1:670a (Abstr.)

56. Baumann, J. E., P. B. Persson, H. Ehmke, B. Nafzak, and H. R. Kirchheim. 1992. Role of endothelium-derived relaxing factor in renal autoregulation in conscious dogs. Am. J. Physiol. 263:F208-F213.

57. Majid, D. S. A., and L. G: Navar. 1992. Supression of blood flow autoregulation plateau during nitric oxide blockade in canine kidney. Am. J. Physiol. 262:F40-F46.
58. Beierwaltes, W. H., D. H. Sigmon, and O. A. Carretero. 1992. Endothelium modulates renal blood flow but not autoregulation. Am. J. Physiol. 262:F943F949.

59. Ito, S., and Y. Ren. 1983. Evidence for the role of nitric oxide in macula densa control of glomerular hemodynamics. J. Clin. Invest. 92:1093-1098.

60. Wilcox, C. S., W. J. Welch, F. Murad, S. T. Gross, G. Taylor, R. Levi, and H. H. H. W. Schmidt. 1992. Nitric oxide synthase in macula densa regulates glomerular capillary pressure. Proc. Natl. Acad. Sci. USA. 89:11993-11997.

61. DeWardener, H. E., R. R. McSwiney, and B. D. Miles. 1951. Renal hemodynamics in primary polycythemia. Lancet. 2:204-206.

62. Wilcox, C. S., J. Payne, and B. D. W. Harrison. 1982. Renal function in patients with chronic hypoxaemia and cor pulmonale following reversal of polycythemia. Nephron. 30:173-177.

63. Nashat, F. S., F. R. Scholfield, J. W. Tappin, and C. S. Wilcox. 1969. The effect of changes in haematocrit on the intrarenal distribution of blood flow in the dog's kidney. J. Physiol. (Lond.). 201:639-655.

64. Wilcox, C. S., X. Deng, A. H. Doll, H. Snellen, and W. J. Welch. 1993. Nitric oxide mediates renal vasodilation during erythropoietin-induced polycythemia. Kidney Int. 44:430-435. 